\section{G210 HEALTH AND EDUCATION WORKING IN COLLABORATION TO REDUCE EMERGENCY DEPARTMENT ATTENDANCES THROUGH ENGAGING WITH CHILDREN ABOUT THE NHS AND HOW TO USE IT}

doi:10.1136/archdischild-2013-304107.222

1,2M Clements, ${ }^{1} \mathrm{~K}$ Evans, ${ }^{2} \mathrm{H}$ Sadler. ${ }^{1}$ Children and Young People Programme, NHS Institute for Innovation and Improvement, Coventry, UK; '2Department of Paediatrics, West Suffolk Hospital NHS Foundation Trust, Bury St Edmunds, UK; ${ }^{3} \mathrm{AhHa}$ Publications Limited, Eastbourne, UK

Aim To work collaboratively with education to produce a lesson planner and teacher resource pack for use in primary schools to teach children about the NHS and how to stay healthy.

To ensure children are seen in the right place at the right time and avoid unnecessary Emergency attendances.

Methods Children and Young people (CYP) feedback that no-one tells them about the NHS until until they are unwell. Nationally there is increased attendance at emergency departments (ED). It is estimated that $40 \%$ of ED attendances could be avoided.

A lesson planner for 11-14yr olds was developed collaboratively with young people in 2010 to teach them about the NHS and distributed to 4000 secondary schools.

Subsequently there has been a demand for a primary care version (see figure 1 - NHS Emergency and Urgent Care Services). This has been co-produced with health, a parent and teacher and graphic designer.

It uses a Monkey puppet and an NHS Monkey song. There are a variety of games for example picture scenario cards and role-play and a series of film clips illustrating different NHS services. E.g. Community Children's Nurses, pharmacists, GP's etc.
Children and young people have been involved in producing and testing the package in mainstream and special schools.

Results The secondary care lesson planner evaluation demonstrated that following education young people were more likely to opt for self- care or primary care.

The primary school lesson planner will be distributed to 19,000 primary schools

It is a high quality interactive, innovative multi-media resource which has been tested and well received in a number of schools and early evaluation and feedback is positive. The teachers resource pack can be picked up and used. It complements the national curriculum across 6 year groups focusing on personal health and social education but also extending into other areas.

Conclusion This is a highly innovative and collaborative piece of work aimed at educating children, young people and families

If this resource prevents just $1 \mathrm{ED}$ attendance per primary school it could save $£ 1.4$ million pound ( $£ 1.2$ million return on investment) but even more importantly it is teaching CYP and families to use the right service at the right time.

\section{British Association for Community Child Health/Child Protection Special Interest Group}

\section{G211 CHILDREN'S TELEVISION VIEWING AND COGNITIVE OUTCOME AT SEVEN YEARS - HELP OR HINDRANCE?}

doi:10.1136/archdischild-2013-304107.223

'S Sivakumaran, ${ }^{2} \mathrm{~S}$ Hope, ${ }^{2} \mathrm{H}$ Bedford, 'J Ellis. 'Paediatrics, Homerton University Hospital NHS Trust, London, UK; ${ }^{2}$ Epidemiology, UCL Institute for Child Health, London, UK

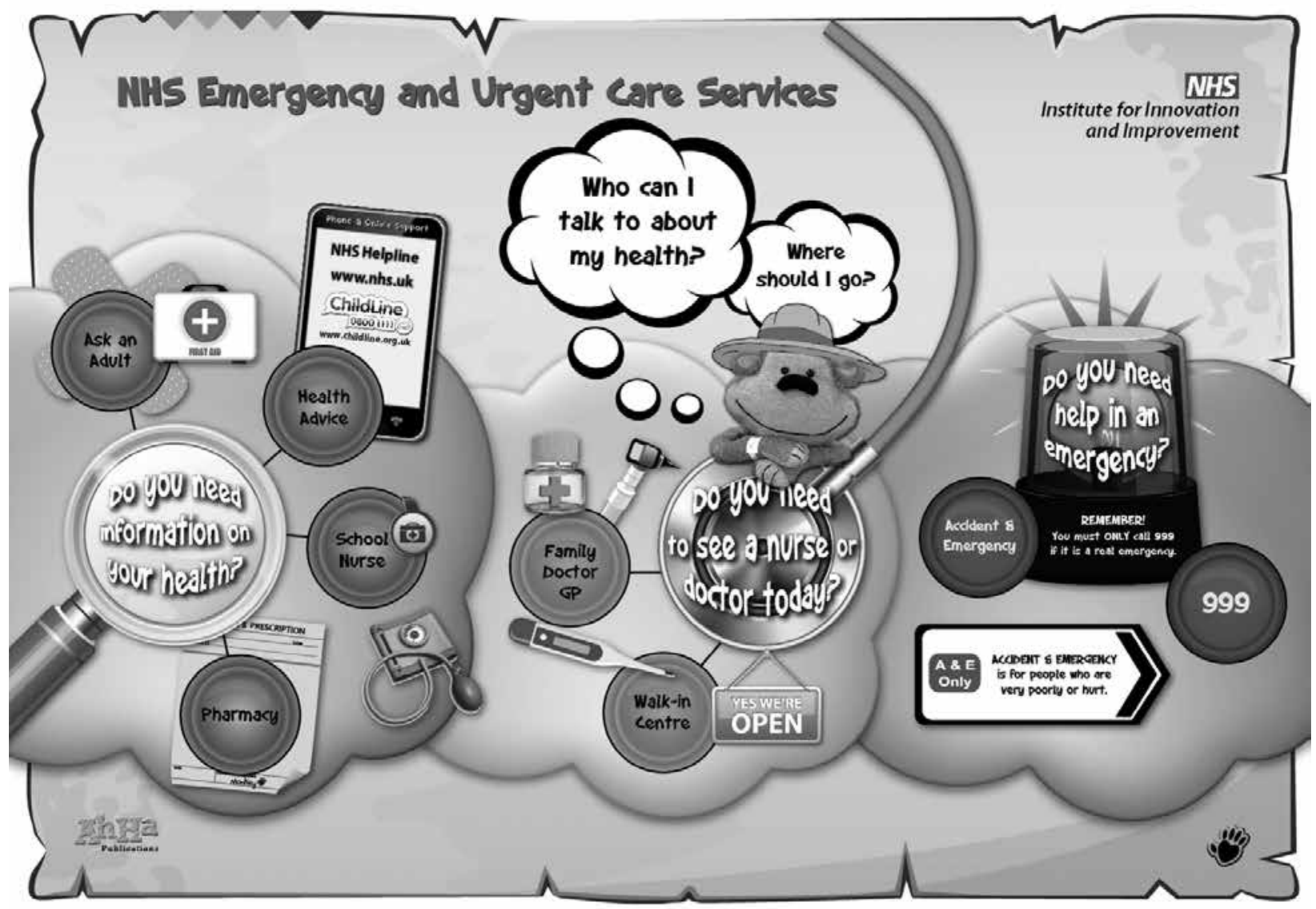


Objective There is conflicting evidence on the association between television viewing in childhood and cognition. No recent UK and few worldwide studies have looked at the longer-term effects of television viewing in childhood. This study investigates the association between verbal and non-verbal cognitive outcomes at 7 years and television habits at 3,5 and 7 years.

Method The study comprises longitudinal and cross-sectional analysis of the United Kingdom Millennium Cohort Study based on 8,148 children with complete data on variables of interest, maternal reports of television viewing and scores from objective tests of cognitive ability (British Ability Scales Second Edition) collected when cohort members were 3, 5 and 7 years. Mean ability scores were converted into the equivalent progress expected in a child at each age group using existing age equivalents derived for the cohort study population. Linear regression was used to estimate the relationship between each subscale and categories of television viewing (in relation to a reference group who watched between 1-3 hours of television a weekday) after adjustment for co-variates.

Results Children who did not watch television at 3 years had verbal ability scores at 7 years approximately 7 months behind the reference group ( $p<0.05$ ); their non-verbal ability skills were approximately 18 months delayed $(p<0.05)$. Children who watched less than 1 hour a weekday had delayed non-verbal skills of approximately 2 months ( $<<0.05)$

There was no significant association between television viewing at 5 and verbal or non-verbal ability at 7 years.

Children who did not watch television at 7 years had verbal ability scores approximately 3 months ahead of the reference group $(p<0.05)$. Those who watched less than 1 hour a weekday were approximately 1 month ahead $(p<0.05$ ). Children who watched over 3 hours of television a weekday had delays of more than 1 month $(p<0.05)$. There was no significant association between television viewing at 7years and non-verbal ability.

Conclusion There was a positive association between television viewing at 3 years and verbal and non-verbal cognition at 7 years in this cohort but a negative association between television viewing at 7 years and verbal skills at 7 years. This may influence potential guidance on television viewing. Possible explanations are the type of programmes watched or accumulation of viewing hours.

\section{G212 PAEDIATRIC ASSESSMENTS IN CHILD PROTECTION: HOW CAN WE MEASURE QUALITY?}

doi:10.1136/archdischild-2013-304107.224

1,2R Crowley, 1,2M Hyrapetian, 1,2E Day, 'D Hodes. 'Community Child Health, NHS Camden Provider Services, London, UK; ${ }^{2}$ School of Paediatrics, London Deanery, London, UK

Aims Child Protection is behind other paediatric specialties in the development of tools to measure the quality of services. We therefore aimed to develop quality indicators in Child Protection that could be used to evaluate paediatric assessments. This study shows how such tools were applied in two Child Protection services and how the resulting outcome data was used to guide service improvement.

Methods A menu of quality measures for paediatric assessment in child protection was formulated from the available guidance and evidence base. Indicators selected by the local team were incorporated into the existing paediatric assessment proforma used for children with suspected maltreatment. The indicators were chosen to be meaningful for the service and to include process as well as outcome measures, without being time-consuming for clinicians. Local teaching sessions informed all doctors about the project and administrators prospectively extracted data for collation in a central database.

Results 84 proformas completed between March and June 2012 were analysed using monthly run charts. Three quality indicators largely within the doctors' control - whether the child was offered a chance to speak to the doctor alone, choose who was present during examination, and attend with a parent or carer - showed some trend towards improvement, which might be expected as a consequence of the 'checklist' function of the proforma. By contrast, the proportion of children whose strategy meeting was attended by a doctor - which requires robust interagency communication in addition to individual action - showed no improvement. Freetext boxes gave qualitative information that has stimulated service improvement, such as ensuring access to a camera to record suspected physical maltreatment. Evidence of ambiguity in one question and suboptimal rates of questionnaire completion have led to modifications in the current cycle of data collection in 2013, the results of which will also be presented and compared.

Conclusions The quality of paediatric assessments can be measured through a clinician-led initiative. We demonstrated that measurement directly stimulates change, particularly when checklists are employed, and can identify specific areas for service improvement. This strategy could be used in other regions and in other areas of child protection.

\section{G213 HOW DOES THE PATTERN OF BRUISING RELATE TO THE MECHANISM OF INJURY IN CHILDREN?}

doi:10.1136/archdischild-2013-304107.225

RE Watson, S Maguire, A Kemp. Institute of Primary Care and Public Health, Cardiff University, Cardiff, UK

Objective It is essential that paediatricians assessing a child with bruising in suspected physical abuse can identify when the explanation for the bruise is implausible. Several published studies describe bruising patterns in populations of abused and non-abused children but we are not aware of any that describe the relationship between mechanism of injury and bruise pattern.

Methods Details of the mechanism of unintentional injury, the number of bruises, location and size were recorded for: children aged 0-11 years attending a Children's Emergency Department (ED) at a tertiary centre and children in the community (4-11 years at school, 0-4 year olds at two nurseries) with bruises of known cause. Students T-test, Chi-squared and Fisher's exact test were used to analyse associations between mechanisms and bruise patterns.

Results 351 children were recruited. Nine mechanisms of injury were recorded (Table 1). 81\% ( $p<0.001$ ) of non-ambulant children (mean age $=9.9$ months, $\mathrm{SD}=6.3$ ) sustained impact injuries whilst $60 \%$ of ambulant children (mean age $=41.7$ months, $\mathrm{SD}=30.4$ ) fell from $<1 \mathrm{~m}$ or standing height $(\mathrm{p}<0.001)$. The majority of children presented with small (mean size $=17.4 \mathrm{~mm}$, range $=2-115 \mathrm{~mm}$ (ED), $1-75 \mathrm{~mm}$ (Community)), and single (86.9\%) bruises related to each incident.

Falls from $<1 \mathrm{~m}$, falls from standing height hitting an object and impact injuries accounted for $79.5 \%$ (279/351) of all incidents. Bruises were most commonly found on the forehead ( $\mathrm{n}=114$, $29.7 \%$ ) knees and shins ( $\mathrm{n}=88,22.9 \%$ ) and head $(\mathrm{n}=39,10.2 \%)$ whilst bruising to certain areas were rare e.g. the front trunk (1.3\%), back of thigh $(0.9 \%)$ and genitalia $(0.3 \%)$.

Conclusion The data demonstrate predominant injury mechanisms for unintentional injury in children that relate to child's level of development. Multiple bruises from a single incident were rare, bruises were small and predominantly to the forehead, knees and shins and head. This predictable pattern of unintentional bruising can aid clinicians to judge the likelihood that a pattern of bruising is consistent with the mechanism of injury, and improve the detection of physical abuse where bruising pattern does NOT fit with a proposed mechanism. 